\title{
Regression of carotid plaques in individuals at low-to-intermediate cardiovascular risk treated with Citozym and Propulzym
}

\author{
Torricelli Piera ${ }^{1}$, Ferorelli Pasquale ${ }^{2}$, De Martino Angelo ${ }^{2}$, Antonelli Francesco ${ }^{2}$, \\ Shevchenko Anna ${ }^{3}$, Beninati Simone, ${ }^{2, *}$ \\ ${ }^{1}$ Surgical Department, Hospital “A. Cardarelli”, Campobasso, Italy \\ ${ }^{2}$ Department of Biology, University of Rome, Tor Vergata, Roma, Italy \\ ${ }^{3}$ Department of Science, People's Friendship University of Russia, Moscow, Russia
}

Email address:

beninati@bio.uniroma2.it (B. Simone)

To cite this article:

Torricelli Piera, Ferorelli Pasquale, De Martino Angelo, Antonelli Francesco, Shevchenko Anna, Beninati Simone. Regression of Carotid Plaques in Individuals at Low-to-intermediate Cardiovascular Risk Treated with Citozym and Propulzym. European Journal of Preventive Medicine. Vol. 2, No. 3, 2014, pp. 33-37. doi: 10.11648/j.ejpm.20140203.12

\begin{abstract}
A prospective trial was performed to assess coronary plaque regression following about 5 months of treatments with two dietary supplements, Citozym and Propulzym. Coronary segments that included the most diseased plaque of 20 selected patients were analyzed using carotid ultrasound (CDUS). Inclusion criteria were as follows: 20 subjects (10 of control group and 10 of test group) aged between 54 and 74 years with a clear diagnosis of carotid plaque and stenosis percentage of not less than $40 \%$ and not more than $70 \%$. The data obtained showed a wide inter-individual variability, with a range of reduction from $4 \%$ to $27 \%$. It is interesting to note that the plaque reduction in one patient reached a value of about $27 \%$ observable with the ultrasonic examination. Since it is clear that carotid plaque morphology/stability is one of the most important factor regarding the definition of cardiovascular risk, our preliminary results suggest the possibility of a non-invasive treatment of carotid plaque.
\end{abstract}

Keywords: Dietary Supplements, Coronary Plaque, Carotid Ultrasound, Atherosclerosis

\section{Introduction}

The carotid artery runs in the neck and divides into the external carotid artery, which vascularizes the face, and in the internal carotid artery that carries blood to the brain. The bifurcation is the most common site of pathology, especially pathologic material accumulate as fat (lipids and cholesterol), calcium, cell debris, leading to the formation of plaques with the consequent progressive narrowing of the artery called stenosis. A stenosis at the level of the carotid arteries due to a deficient supply of blood to the brain. The most important risk of the presence of carotid plaques is the erosion of the surface of the plate with the fragmentation of particles that go to occlude terminal arteries of the brain, causing cerebral ischemia and stroke. The stroke can be massive and therefore lethal or marginal. Everything depends on the extent of the brain areas affected. The reversible stroke is called "transient ischemic attack" (TIA), regressing within a few hours. These events are usually an alarm bell, if not correctly interpreted and treated can lead to a permanent stroke called "cerebral vascular accident" (CVA). The symptoms that occur most frequently are: paralysis of an arm and/or leg, sudden loss of vision, inability to speak properly for a few minutes. The most common risk factors are: smoking, diabetes, hypertension, obesity, hypercholesterolemia, and age. Treatment may be surgery (endarterectomy) or medical (antiplatelet therapy). Randomized multicenter studies have shown that in asymptomatic patients surgical treatment is indicated when the stenosis reaches $70 \%, 50 \%$ in symptomatic patients. Before the operation is carried out a mapping Ecocolor Doppler of the carotid bifurcation that can significantly reduce the extent of the incision; thus obtaining an acceleration of healing time. Surgical treatment is performed under general anesthesia and involves complete removal of the carotid plaque. It has been shown that a percentage ranging from $20-50 \%$ of the TIA is due to carotid disease. 
Then through the execution of a color Doppler of the carotids, fast examination and non-invasive, it is perfectly possible to diagnose the presence of a carotid plaque, its morphology and the percentage of stenosis. If a person is part of a category and then a risk, it is important to run an Ecocolor Doppler every 12 months [1]. The active ingredient used in the longest antiplatelet therapy for the treatment of atherosclerosis has been acetylsalicylic acid. Aspirin exerts its antiplatelet effects by acetylating platelet cyclooxygenase, thus irreversibly inhibiting the formation of thromboxane in platelets [2]. Recently, folic acid, the vitamin B group, vitamin $\mathrm{C}$ and $\mathrm{D}$, have been identified as appropriate supplements to reduce the negative effects of homocysteine, which facilitates the formation of thrombi in arteries and veins [3]. Free radical excess and oxidative stress are implicated in the formation and progression of atherosclerotic plaque through actions on susceptible vascular cells, such as by activating xanthine oxidase. Purine bases and other antioxidant compounds could play important protective roles in atherogenesis, as could non enzymatic low molecular weight thiol defenses, not previously evaluated in carotid artery plaque [4,5]. The formation of foam cells from monocyte-derived macrophages involves the uptake of modified lipoproteins by scavenger receptors. Antioxidants inhibit lipoprotein oxidation and may also modulate gene expression. The inhibitory effect of $\mathrm{N}$-acetylcysteine (NAC) on atherosclerotic processes has been reported [6]. NAC express its antioxidant activity on the expression of the class A scavenger receptor (SR-A) types I and II in human macrophages. NAC leads to a decrease in SR-A mRNA and initially also to an attenuated uptake of modified lipoproteins [7]. Glutathione is an endogenous antioxidant and has an ubiquitous role in many of the body's defenses. Treatment with NAC has been shown to increase levels of glutathione. NAC has been proposed as an antiatherogenic agent [8].

Low-density lipoprotein (LDL) oxidation is a potential atherogenic agent, and protecting LDL from oxidation prevents atherogenesis. It has been shown that L-aspartate and L-glutamate decrease lipid peroxidation after reoxygenation by means of the initiation of the cardiopulmonary bypass circuit (CPB), when supplemented to the $\mathrm{CPB}$ prime, and so they may protect against atherogenesis [9]. Calcification at vascular sites is the most threatening localization and manifests as part of atherosclerosis or arteriosclerosis. Atherosclerosis is often accompanied by intimal plaque calcification, whereas arteriosclerosis is characterized by calcification of the media. The severity of calcification of cerebral or coronary atherosclerotic plaques is associated with an increased incidence of events such as stroke or myocardial infarction [10]. Furthermore, the effect of citric acid and certain carbonates of alkali metals on hypercholesterolemia and atherosclerosis has been studied with results that have been shown to reduce the formation of carotid plaques $[11,12]$. On the basis of this information, the aim of our project was to evaluate the action of two dietary supplements: Citozym
(CYZ) rich in vitamins $\mathrm{B}, \mathrm{C}$ and $\mathrm{D}$, and Propulzym (PRZ), rich in NAC, glutamic acid, calcium carbonate and citric acid, on the carotid plaques, in individuals at low-to-intermediate cardiovascular risk.

\section{Material and Methods}

\subsection{Subjects and Treatments}

Members of this study were recruited from the ambulatory cardiac clinic and all the patients gave informed consent. Inclusion criteria were as follows: 20 subjects aged between 54 and 74 years with a clear diagnosis of carotid plaque and stenosis percentage of not less than $40 \%$ and not more than $70 \%$, were examined for a period of about five months. Members of control group (n.10) were not treated while patients of the test group (n.10) were daily orally treated with CYZ and PRZ (Citozeatec S.r.l. Peschiera Borromeo, Milano, Italy). The extent of carotid plaques in control and test groups was assessed by means of carotid ultrasound Doppler Ecocolor (MC-DU-iVis30 CE/FDA), carried out once a month (Figure 1). During the trial, the hematological data of patients (cholesterol, HDL and LDL, blood glucose, blood urea nitrogen, creatinine and uric acid) were collected monthly (data not shown).

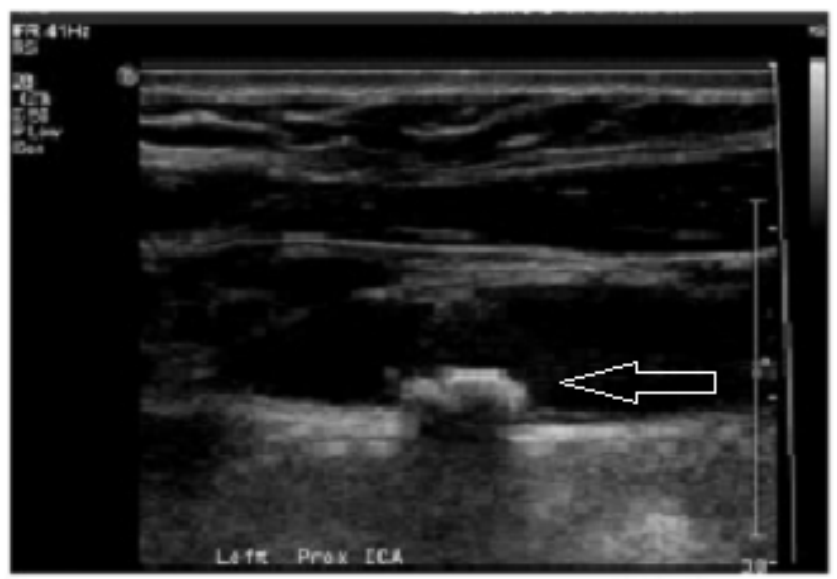

Figure 1. Representative carotid artery ultrasonography of a patient showing the presence of a plaque with obstruction assessed at around $30 \%$

The treatment was carried out according to the following protocol: in the absence of contraindications to the two supplements they were administered in increasing doses during the planned 150 days of treatment. Therefore, $9 \mathrm{~g}$ of CYZ every morning during breakfast for all the period of treatments; $9 \mathrm{~g}$ of CYZ for two days at dinner, increased to $18 \mathrm{~g}$ for the next 7 days and then $40 \mathrm{~g}$ until the end of treatment; finally, $26 \mathrm{~g}$ of CYZ for lunch, from the $21 \mathrm{st}$ to the end of treatment. PRZ was administered in the dose of $6.5 \mathrm{~g}$ from the eleventh day of treatment until the twentieth, and then increased to $13 \mathrm{~g}$ from the twenty-first day until the end of the treatment to dinner, together with $6.5 \mathrm{~g}$ during lunch. An accurate observation of possible adverse effects on the patient was performed. 


\subsection{Statistical Analysis}

All results are expressed as median values and interquartile range. Data analysis software system, (version 9.0) from StatSoft (2009, www.statsoft.com) was used for the statistical analysis. For analyses of independent groups the Student $t$-test (for variables with normal distribution) and Mann-Whitney's U test (for variables with non-parametric distribution) were carried out. For normal distribution, variables were analyzed by the Kolmogorov-Smirnov test with Lilliefors correction. Correlation between analyzed parameters was estimated by Pearson's test (for variables with normal distribution), and Spearman's test (for variables with non-parametric distribution). Variables describing clinical parameters (age, sex, present smoking, presence of diabetes mellitus, dyslipidemia and hypertension) were entered into a multivariate regression analysis to determine independent predictors of parameters describing the pathological status of patients.

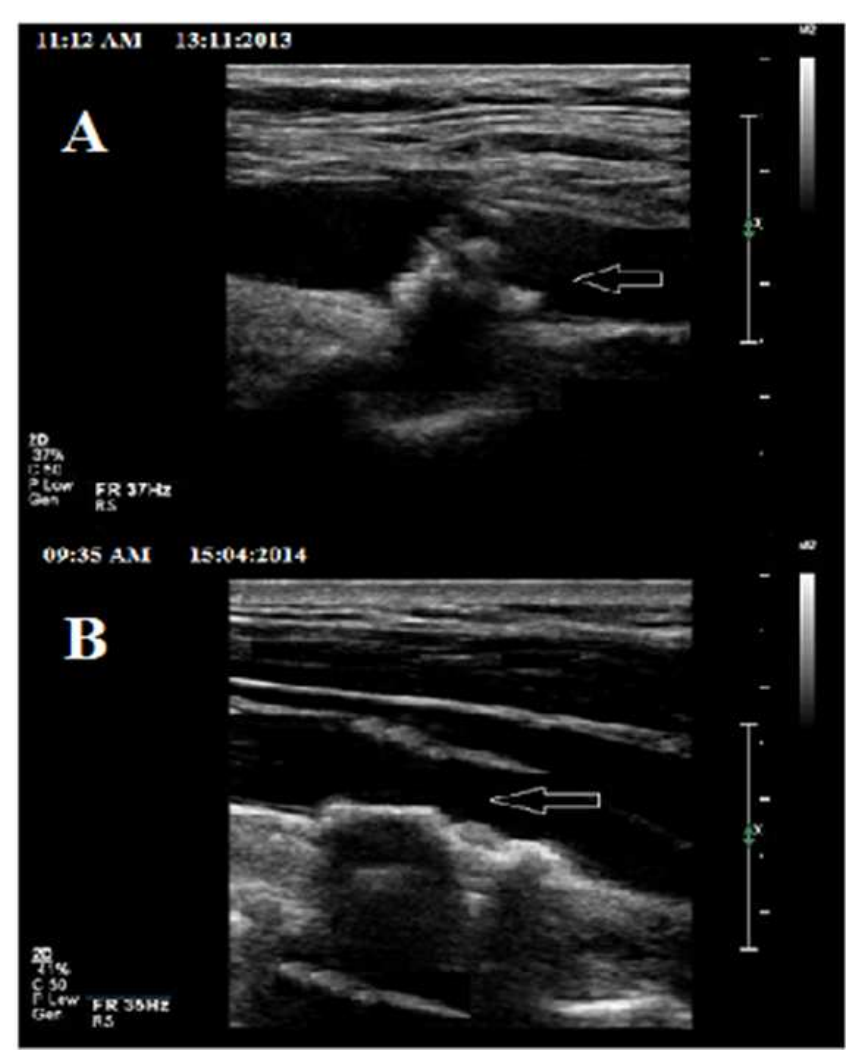

Figure 2. A. Ultrasonography of the carotid artery of the patient n.8 (R.O.), showing the presence of a plaque with obstruction assessed at around $55 \%$. $\boldsymbol{B}$. plaque reduction of about $27 \%$ after 150 days of treatment with the combination of CYZ and PRZ (see Material and Methods).

\section{Results}

Seven of the 10 plaques investigated were found to be free from symptoms, while the remaining three were found to be symptomatic following expert specialist stroke physician assessment. The mean age of the symptomatic patients was 70 years compared with 62 years for the asymptomatic None of the patient characteristics: sex (10 males), current or past tobacco smoking $(60 \%)$, hypertension $(60 \%)$, hypercholesterolemia $(60 \%)$, diabetes mellitus $(30 \%)$, ischemic heart disease $(30 \%)$, family history of stroke $(40 \%)$, alcohol consumption (40\%) and peripheral vascular disease (10\%), had a statistically significant relationship to the presence of symptoms $(p>0.05)$. In Table I is depicted the effect of the treatment of patients according to the protocol reported in Material and Methods. Data show a rather marked individual variability, with a range of reduction from $4 \%$ to $27 \%$ (\% Red). Three cases showed a lower reduction (patients no. 3,5 and 10), this observation may suggest a potential sign of stabilization of plaques.

Table 1. Percentages of reduction of carotid plaques in patients treated with $C Y Z$ and PRZ with the protocol described in Material and Methods. The initial values represent the average of two ultrasound assessments. Data reported in "\% Final stenosis" were extrapolated from 5 ultrasound examinations and are the mean of five independent results. $(\%$ Red: percentage of reduction).

\begin{tabular}{lllllll}
\hline Patient & Age & $\begin{array}{l}\text { \% Initial } \\
\text { Stenosis }\end{array}$ & mean1 & $\begin{array}{l}\text { \% Final } \\
\text { Stenosis }\end{array}$ & mean2 & \%Red \\
\hline 1 E.N. & 65 & $40 / 50$ & 45 & $35 / 40$ & 37 & 18 \\
2 T.S. & 57 & $40 / 50$ & 45 & $35 / 40$ & 37 & 18 \\
3 H.G. & 66 & $60 / 60$ & 60 & $50 / 60$ & 55 & 8 \\
4 T.D. & 54 & $40 / 40$ & 40 & $30 / 35$ & 33 & 18 \\
5 F.C. & 74 & $70 / 70$ & 70 & $70 / 65$ & 68 & 4 \\
6 R.G. & 68 & $50 / 50$ & 50 & $45 / 45$ & 45 & 10 \\
7 R.V. & 57 & $40 / 50$ & 45 & $30 / 40$ & 35 & 13 \\
8 R.O. & 66 & $50 / 60$ & 55 & $40 / 40$ & 40 & 27 \\
9. P.F. & 70 & $60 / 70$ & 65 & $55 / 60$ & 57 & 12 \\
10 G.H. & 65 & $50 / 55$ & 52 & $45 / 50$ & 48 & 9 \\
\hline
\end{tabular}

It is also evident that in some cases it has come to a reduction of $27 \%$ (patient no. 8 ) with a clear change in the volume of the plaque (Figure 2). Furthermore, we also observed significant values of carotid plaque reduction of 18\% (patient no. 1,2 and 4). Since ultrasound evaluation does not allow to assess accurately the percentage of carotid stenosis, we considered the means of two ultrasound readings (mean 2) at the end of treatment and compared these values with the initial data (mean 1) obtained prior the treatment. The treatment was performed in patients with abnormal blood pressure and cholesterol levels in excess of $20 \%$ from normal values. Taking medication and diet were considered during the drafting of the natural history of each subject.

\section{Discussion}

There are multiple research data that led to the recognition that atherosclerosis is a chronic inflammatory condition which is characterized by the formation of fibrotic leukocytes containing plaque $[13,14]$. The association between inflammation and oxidative stress is well documented with a number of studies of inflammatory conditions reporting elevated levels of 8-oxo-7, 8-dihydro-2'-deoxyguanosine (8-oxodG), one of the oxidative stress biomarker [15] . the inflammatory response can lead to the recruitment of activated leukocytes, which 
may, in turn, give rise to a "respiratory burst" — an increased oxygen uptake that causes the release of high quantities of reactive oxygen species (ROS), such as superoxide and hydrogen peroxide, with possible subsequent DNA damage production [16]. Proof of this proposal comes from the report of Dizdaroglu et al., who demonstrated that exposure to activated leukocytes caused DNA base modifications in human cells typical of those induced by hydroxyl radical attack [17]. Moreover, oxidatively damaged DNA has been found in atherosclerotic lesions and leukocytes of atherosclerotic patients [18,19,20]. Furthermore, there are data which provide evidence that oxidatively induced damage not only occurs in the DNA of atherosclerotic patients but also has some influence on long-term clinical outcome and a recent study demonstrated interesting evidence concerning direct/active involvement of oxidatively damaged DNA/failure of DNA repair in atherosclerosis, in an animal model [21,22]. It is believed that carotid plaque morphology/stability is one of the most important factors regarding the definition of cardiovascular risk $[23,24]$. However, there are conflicting data concerning factors which influence the plaque stability [25]. It is an important issue since it has become increasingly evident that the plaque morphology may be responsible for its rupture which in turn may give rise to an acute clinical outcome. It is also possible that plaque morphology may be shaped by oxidative stress [26]. Our experimental processing, carried out following a protocol that uses two types of dietary supplements (CYZ and PRZ) according to a precise dosage of administration (see Material and Methods) for 150 days, led to a clear reduction in carotid plaques in patients with an initial stenosis between $40 \%$ and $70 \%$. These results suggest that daily use of the two supplements mentioned can probably increase the blood circulation in the brain, improving cognitive skills of the patient and possibly reducing the risk of cerebral vascular disease events. The wider distribution and the abundant presence of antioxidants in the CYZ and PRN formulation, together with the present results, may suggest that these mixture of antioxidants may have the potentiality of a possible contribute to the improvement of the quality of life of individuals affected of carotid atherosclerosis, at low-to-intermediate cardiovascular risk

\section{Conflict of Interest}

The authors have no potential conflicts of interest.

\section{References}

[1] Johnston SC, Rothwell PM, Nguyen-Huynh MN, et al, Validation and refinement of scores to predict very early stroke risk after transient ischaemic attack in Lancet, 2007;369, no $9558,283-292$.

[2] Lewis, H. D.; Davis, J. W.; Archibald, D. G.; Steinke, W. E.; Smitherman, T. C.; Doherty Je, J. E.; Schnaper, H. W.; Lewinter, M. M.; Linares, E.; Pouget, J. M.; Sabharwal, S. C.;
Chesler, E.; Demots, H. Protective Effects of Aspirin against Acute Myocardial Infarction and Death in Men with Unstable Angina". New England Journal of Medicine 1983;309 (7),396-403.

[3] Nygård, O; Vollset, SE; Refsum, H; Stensvold, I; Tverdal, A; Nordrehaug, JE; Ueland, M; Kvåle, G Total plasma homocysteine and cardiovascular risk profile. The Hordaland Homocysteine Study.". JAMA : the journal of the American Medical Association 1995;274 (19): 1526-1533.

[4] Shin MH, Moon YJ, Seo JE, Lee Y, Kim KH, Chung JH Reactive oxygen species produced by NADPH oxidase, xanthine oxidase, and mitochondrial electron transport system mediate heat shock-induced MMP-1 and MMP-9 expression. Free Radic Biol Med. 2008;44(4):635-645

[5] Ciari I., Terzuoli L., Porcelli B., Coppola M G, Marinello E. Antioxidant status and purine bases in carotid artery plaque. Nucleosides Nucleotides \& amp Nucleic Acids. 2008; 27(6):624-627

[6] Meng XP, Yin CS, Cui JH, Li ZX, Wang L, Wang YW, Li YL. Inhibitory effect of $\mathrm{N}$-acetylcysteine upon atherosclerotic processes in rabbit carotid. Zhonghua Yi Xue Za Zhi. 2009; 89 (26):1850-3

[7] Svensson L, Norén K, Wiklund O, Lindmark H, Ohlsson B, Hultén LM Inhibitory effects of $\mathrm{N}$-acetylcysteine on scavenger receptor class A expression in human macrophages. J Intern Med. 2002; 251(5):437-446

[8] Dodd S. Dean O. Copolov DL. Malhi GS. Berk M. $\mathrm{N}$-acetylcysteine for antioxidant therapy: pharmacology and clinical utility in Expert Opinion on Biological Therapy, vol. $8, \mathrm{n}^{\mathrm{o}} 12,2008$, pp. 1955-1962

[9] Yanni AE, Yatzidis HA, Kavantzas NG, Agapitos EV, Perrea DN, Karayannacos PE. Dietary L-aspartate and L-glutamate inhibit fatty streak initiation in cholesterol-fed rabbit. Nutr Metab Cardiovasc Dis. 2003;13(2):80-86

[10] Schlieper G, Westenfeld R, Brandenburg V, Ketteler M. Inhibitors of calcification in blood and urine. Semin Dial. 2007;20(2):113-121

[11] Kaĭdin DA. The effect of citric acid on experimental hypercholesteremia in atherosclerosis in rabbits. Biull Eksp Biol Med. 1968;65(3):36-39

[12] Kă̌din DA, Grebennik LI. The effect of citric acid and alkali and alkaline earth metal carbonates on the development of experimental hypercholesteremia and atherosclerosis in rabbits. Kardiologiia. 1967;7(4):123-127.

[13] Libby P. Inflammation in atherosclerosis Nature, 2002; $420,868-874$

[14] Ross R. Atherosclerosis - an inflammatory disease N Engl J Med, 1999;40,115-126

[15] Wiseman H, Halliwel B. Damage to DNA by reactive oxygen and nitrogen species: role in inflammatory disease and progression to cancer Biochem J, 1996;313(1) 17-29

[16] Cooke MS, Olinski R, Evans MD. Does measurement of oxidative damage to DNA have clinical significance? Clin Chim Acta, 2006; 365,30-49

[17] Dizdaroglu M, Olinski R, Doroshow JH, Akman SA Modification of DNA bases in chromatin of intact target human cells by activated human polymorphonuclear leukocytes Cancer Res, 1993;53,1269-1272 
[18] De Flora S, Izzotti A, Walsh D, Degan P, Petrilli GL, Lewtas J Molecular epidemiology of atherosclerosis FASEB J, 1997;11,1021-1031

[19] Collins AR, Gedik CM, Olmedilla B, Southon S, Bellizzi M Oxidative DNA damage measured in human lymphocytes: large differences between sexes and between countries, and correlations with heart disease mortality rates FASEB J, $1998 ; 12,1397-1400$

[20] Martinet W, Knaapen MW, De Meyer GR, Herman AG, Kockx MM Elevated levels of oxidative DNA damage and DNA repair enzymes in human atherosclerotic plaques Circulation, 2002;106,927-932

[21] Izzotti A, Piana A, Minniti G, Vercelli M, Perrone L, De Flora S Survival of atherosclerotic patients as related to oxidative stress and gene polymorphisms Mutat Res, 2007;621,119-128

[22] Mercer JR, Cheng KK, Figg N, Gorenne I, Mahmoudi M, J. Griffin $\mathrm{J}$ et al.DNA damage links mitochondrial dysfunction to atherosclerosis and the metabolic syndrome Circ Res, 2010;107,1021-1031
[23] Honda O, Sugiyama S, Kugiyama K, Fukushima H, S. Nakamura S, S Koide et al.Echolucent carotid plaques predict future coronary events in patients with coronary artery disease J Am Coll Cardiol, 2004;43,1177-1184

[24] Johnsen SH, Mathiesen EB, Joakimsen O, Stensland E, Wilsgaard T, Lochen ML et al.Carotid atherosclerosis is a stronger predictor of myocardial infarction in women than in men: a 6-year follow-up study of 6226 persons: the Tromso Study Stroke, 2007;38,2873-2880

[25] Hellings WE, Peeters W, Moll FL, Piers SR, Van Setten J, Van der Spek PJ et al. Composition of carotid atherosclerotic plaque is associated with cardiovascular outcome: a prognostic study Circulation, 2010;121,1941-1950

[26] Bitto A, De Caridi G, Polito F, Calo M, Irrera N, Altavilla D et al. Evidence for markers of hypoxia and apoptosis in explanted human carotid atherosclerotic plaques J Vasc Surg, 2010;52,1015-1021 